\section{Science popularity stakes}

SIR - You ask "Is molecular biology yet a science?" (Nature 355, 201; 1992). I would answer "No, it's rather a game of molecular roulette". However, the real question is whether we do want molecular biology to become a quantitative science, and relegate it back to the ivory tower, or whether it should acquire 'popular science' status.

Anyone old enough to have witnessed its birth in the early 1960 s can only agree with your characterization of molecular biology as "... well on the way to becoming a largely qualitative science". Every older biochemist, steeped in the law of mass action and other thermodynamic principles applicable to biology, can add other examples for your list. All would show that neglect of quantification in molecular biology may cause important problems to be overlooked.

The new style molecular biology is also helping the literature to mushroom. A plethora of new journals with titles certain to contain elements of any of the words "molecular", "genetic", "biology", "technology" or their permutations has appeared, and established journals are undergoing name changes to keep up. Witness finally the spectacle of learned societies such as the venerable American Society of Biological Chemists undergoing the ultimate humiliation of becoming the American Society of Biological Chemistry and Molecular Biology. How much more molecular is the biology done by these biochemists? place.

\section{J. M. Kaper}

Microbiology and Plant Pathology Laboratory,

Plant Sciences Institute, Beltsville, Maryland 20705, USA

To be sure ". . . the triumphant pervasiveness . . ." of molecular biology new style should also be credited for awakening the public to its promise. Never mind that those awakened are also the politicians, bureaucrats, science managers, conference organizers, and so on, always on the prowl for buzzwords. Thus, the adjective "molecular" has become indispensable to the young biologist seeking to enhance his or her career. The molecules and the biology (and their quantification) unfortunately often take second

But for the future of molecular biology perhaps the best solution would indeed be to let the de-quantification process take its due course, in order that the roulette aspects be allowed to take their predominant place. The defining moment for molecular biology to become a truly 'popular' science would then be its relabelling as 'molecular biotechnology', with "mobi-" or "mobiotech" as popular acronyms for short. That should ultimately result in a kind of 'trickle-up' process and perhaps improve the funding for the purists among us who wish to pursue their law of mass action 'mobiotech-style' to the hilt, and everybody would be happy.

Beltsville Agricultural Research Center,

\title{
Counting on taxonomy
}

SIR - Gaston and May's ${ }^{1}$ estimate of the worldwide distribution of taxonomists among taxa and their bearing on the documentation and conservation of biodiversity can be made more precise for the taxonomists of Spain. Since 1987 we have conducted a continuous survey of Spanish taxonomists providing a census on an automated database system called DIRTAX ${ }^{2,3}$. A total of 1,156 taxonomists has been recorded and their distribution by different categories is given in the tablle.

Strikingly, the percentage of taxonomists working on plants, microorganisms and animals closely fits estimates of US taxonomists. Distribution for lower categories of Kingdom Animalia are less concordant, especially the high number of arthropod taxonomists.

Approximately 60 per cent of registered taxonomists in Spain are professional, and 16 per cent have a temporary status, relying on grants.

The distribution of taxonomists by environment shows a preponderance of
DISTRIBUTION OF SPANISH TAXONOMISTS (\%)

\begin{tabular}{lrlrlr}
\multicolumn{2}{c}{ By kingdom } & \multicolumn{2}{c}{ By environment } & \multicolumn{2}{c}{ By status } \\
Monera & $\mathbf{3 . 6}$ Terrestrial & 53.3 & Professional & $\mathbf{5 6}$ \\
Plantae & $\mathbf{2 8}$ Marine & 16.6 & On grants & $\mathbf{1 6}$ \\
Animalia & $\mathbf{6 8}$ & Freshwater & 10.7 & Amateur & $\mathbf{1 3}$ \\
Tetrapods & $\mathbf{1 3 . 4}$ & Fossils & 10.2 & Other & $\mathbf{1 3}$ \\
Flsh & $\mathbf{7 . 0}$ Parasites & 2.8 & & \\
Arthropods & $\mathbf{4 8 . 2}$ & Subterranean 0.4 & & \\
Other iny & $\mathbf{3 1 . 4}$ & & &
\end{tabular}

terrestrial taxonomists, despite the fact that the highest diversity is in the marine environment. Number of species in a phylum is highly correlated with number of taxonomists in that group, $\left(R^{2}=83.2\right)$. Elisa Bello

\section{Jose M. Becerra}

Antonio G.-Valdecasas

Museo Nacional de Ciencias Naturales,

José Gutierrez Abascal,

2. 28006 Madrid, Spain

1. Gaston, K. L. \& May, R. M. Nature 356, 281-282 (1992)

G. Valdecasas, A., Bello, E., Reyes, J. \& Becerra, J. M. Sci. Software 5, 303-308 (1989).

3. Bello, E. \& G.-Valdecasas. A. in Abstr. 4th int. Congr. Systematic Evol. Biol. (1990)

\section{A new kind of orphan drug?}

SIR - In the United states an Orphan Drug Act was passed in 1983 to give companies incentives in the form of grants, tax credits and a seven-year marketing monopoly for the development of drugs for rare diseases - those that affect fewer than 200,000 people. Meanwhile some of these so-called orphan drugs, for instance aerosol pentamidine, which is used to treat pneumonia caused by Pneumocystis carinii, have made their developers hundreds of millions of dollars'.

We should like to draw attention to a new species of orphan drug: timeproven, essential, standard drugs, unfortunately too cheap from the distributors' point of view to justify further marketing.

In Austria, taeniasis (beef tapeworm) is endemic. Between 1986 and 1991, 82 cases have been verified in an Innsbruck laboratory serving a population of 900,000 . At the time of the diagnosis of taeniasis, approximately 27 per cent of the patients had already undergone inappropriate anthelmintic therapy (mostly mebendazole) without clinical response ${ }^{2}$.

Niclosamide is the drug of choice for Taenia saginata ${ }^{3}$. Since November 1983, niclosamide (Yomesan, Bayer) has no longer been registered in Austria. The company concerned says that the costs of official registration and distribution make continued marketing unjustifiable.

Praziquantel $^{3}$, the only other drug effective in taeniasis, has been registered in Austria only for administration to animals. It appears that only animals promise sufficient consumption of the drug to warrant the costs of official registration and marketing.

This situation seems particularly ironic since niclosamide is listed in the WHO's Model List of Essential Drugs ${ }^{4}$. Perhaps time-proven drugs that are too cheap and needed in too small quantities to ensure continued profit to the pharmaceutical companies should be designated as orphan drugs, to contribute to their more general availability.

\section{F. Allerberger}

M. P. Dierich

Institute for Hygiene,

University of Innsbruck, Innsbruck 6020 .

Austria

1. Gibbons, A. Science 248, 678-679 (1990).

2. Allerberger, F. Mitt. Osterr. Ges. Tropenmed. Parasit. 9. 65-72 (1987).

3. Med. Let. 34, 17-26 (1992)

4. WHO Expert Committee WHO Tech. Rep. Ser. 796 (1990).

5. Gershon, D. Nature 345, 759 (1990) 